\title{
Study on Synchronous Tracking Control with Two Hall Switch-type Sen- sors Based on Programmable Logic Controller
}

\author{
Li Shangrong $^{1,2, *}$, Zhang Chunjie ${ }^{1}$ and Zhang Weiping ${ }^{1}$ \\ ${ }^{I}$ Department of mechatronics, Jiangsu University of Technology, Changzhou 213001, China \\ ${ }^{2}$ Institute of Bin-inspired Structure and Surface Engineering, Nanjing University of Aeronautics and Astronautics, \\ Nanjing 210016, China
}

\begin{abstract}
Positioning control is widely used in many fields of applications, such as CNC machine tools, and robotic motion control. This paper presents a novel method of system control for tracking the moving object by using two switchtype Hall sensors to detect their position offset with programmable logic controller (PLC). The experimental results show that the moving object (Hall switch sensor) could follow the magnets in different positions without departing from each other when the rotating speed of the magnets is no more than $100 \mathrm{rpm}$ and its radius not more than $70 \mathrm{~mm}$.
\end{abstract}

Keywords: FX3U PLC, Hall switch-type sensor, plane interpolation, synchronous tracking control.

\section{INTRODUCTION}

There is a wide range of applications for motion trajectory control in the fields of aerospace, military, robotics, CNC machine tools and automated assembly in industrial production [1-3]. AC servo systems are used as important actuating and control module for automatic equipment, such as robot systems, and CNC machine tools, based on interpolation principle [4-6]. A moving object tracked means which is tracked in real-time synchronization way by another object. It includes finding the moving body first and then tracking it synchronously, which requires the tracking object to have a higher motion performance [7]. The core issue of tracking moving objects is how to obtain the magnitude and direction of the deviation between a moving object and a tracking object, and then adjust the size and direction of the deviation. Some linear displacement sensors, such as linear Hall sensor, could be used to measure the deviation. One linear Hall sensor can only detect the magnitude, therefore, two sensors at least are necessary to achieve the size and direction of the deviation. At the same time, linear displacement sensors need $\mathrm{A} / \mathrm{D}$ module to convert analog signal into digital signal, which is then transferred to the controller $[8,9]$. For some control system without having high precision, two linear sensors and $\mathrm{A} / \mathrm{D}$ module will undoubtedly increase the cost of the system significantly.

In this paper, a tracking control problem, by using a Hall sensor to determine how to accurately track a round magnet with $8 \mathrm{~mm}$ diameter displaced on the circular disc with a certain frequency, is firstly presented. During the tracking process, the head of the Hall switch type sensor is not permitted

*Address correspondence to this author at the Department of mechatronics, Jiangsu University of Technology, Changzhou 213001, China; Tel: 86-0519-86953210; E-mail: jxlsr_jstu@163.com to separate the magnet. Following this, a control implementation scheme including only two Hall switch type sensors used without any linear displacement sensor and A/D module, is presented.

The tracking test system configuration is shown in Fig. (1). In Fig. (1a), the tracked circular magnet $(\Phi 8 \mathrm{~mm})$ is placed on the rotating disc, which is actuated by a PLC controller, an inverter and a three-phase AC motor with a reducer having reduction ratio of 15 . The Hall sensor tracking a magnet is mounted on a workbench actuated by planar two axes feed system controlled by two identical AC servo units. The synchronous tracking state is marked by an indicator light on the end of the Hall sensor. Active movement and tracking movements are implemented as shown in Fig. (1b) . The tracking command information is obtained through HMI (human machine interaction). The active movement is achieved by an inverter to actuate a three-phase AC motor. Following this, the $\mathrm{AC}$ motor is used to drive the disc and the magnet rotates. The tracking movement is realized by a positioning control module to control the two-axis servo drive units in the way of planar interpolation. Fig. (1c) shows the photo of test setup of position tracking system.

\section{MATERIALS AND METHODOLOGY}

The electrical configuration components of the synchronous tracking system mainly consist of a PLC controller (FX3U PLC), a positioning module (FX2N-20GM), a touchscreen (GOT11), an inverter (FR-D720) and two groups servo drive units (MR-J3-10A). The electrical connections between the modules are shown in Fig. (2). As the two axes servo drives were completely identical. Fig. (2) shows only the electrical connection for one feed axis servo drive unit. The magnet's active movement and the Hall sensor's plane interpolation tracking movement in the system are outlined below. 


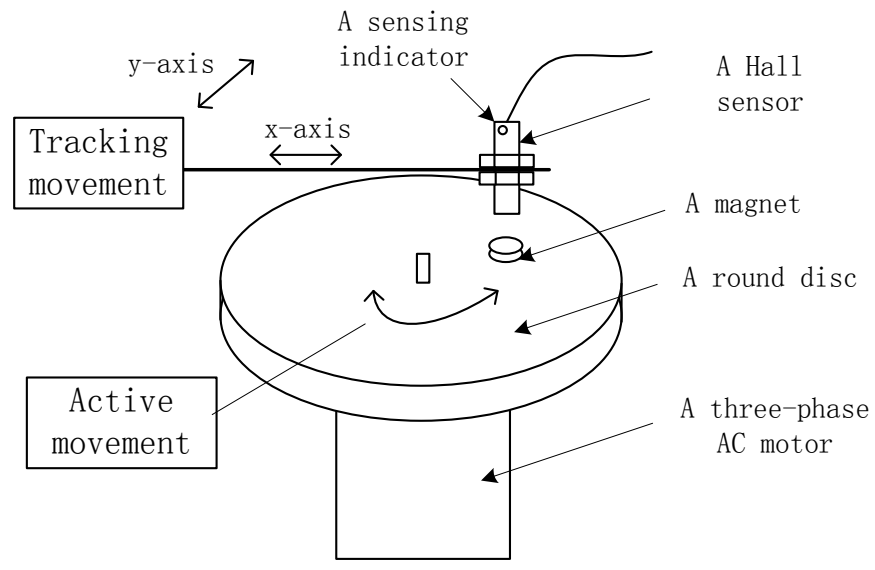

(a) Schematic diagram for a moving magnet tracked by a hall sensor

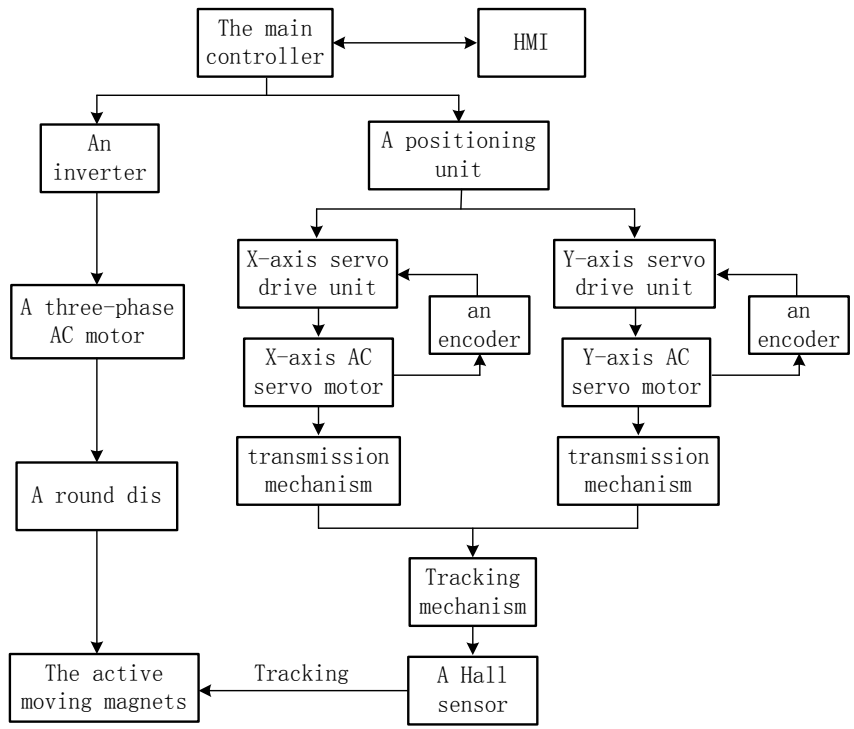

(b) a control scheme for a moving magnet tracked by a Hall sensor

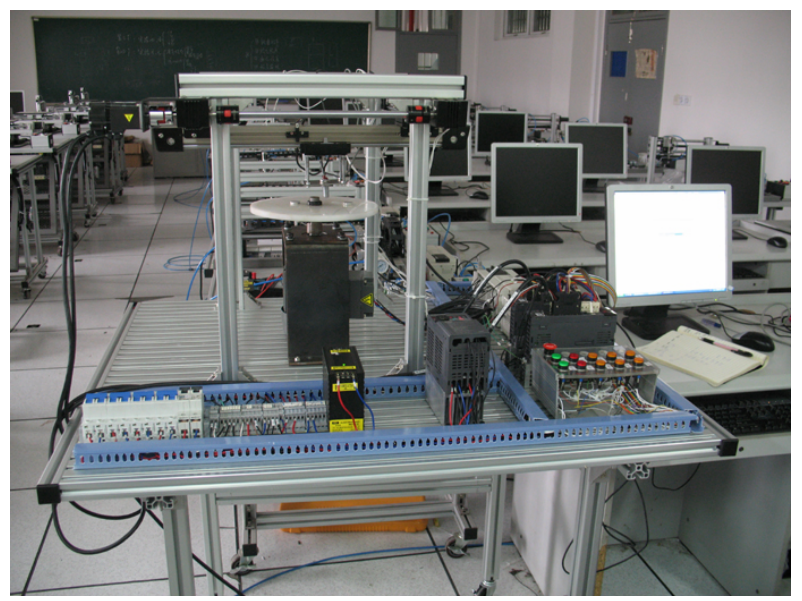

(c) Photo of the position tracking experimental setup

Fig. (1). a tracking control system of an actively rotating magnet tracked by Hall sensors moving in planar interpolation mode. 


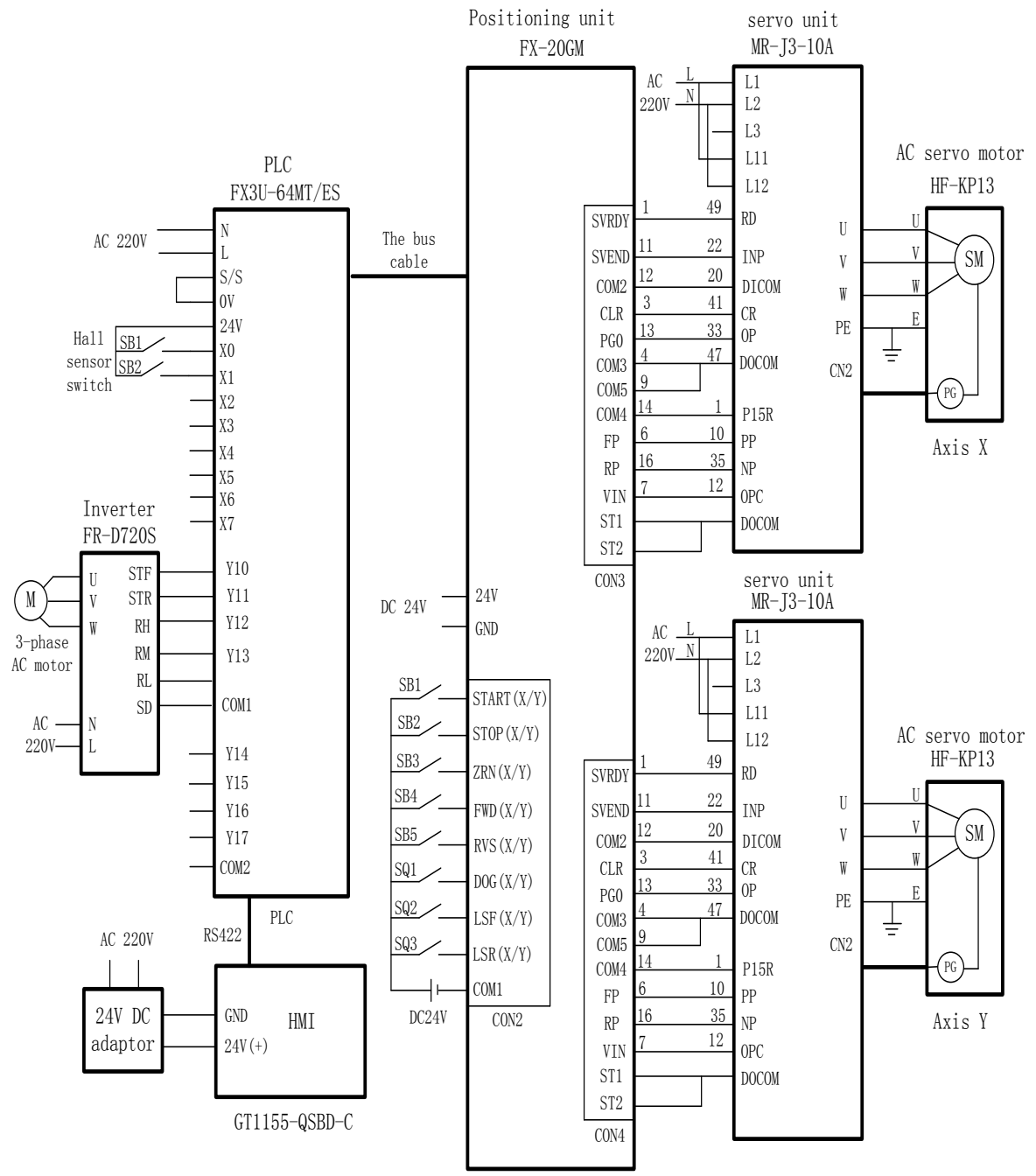

Fig. (2). An electrical schematic for moving object tracked control system.

\subsection{The Active Movement of the Magnet}

The active moving system mainly consists of an inverter, an $\mathrm{AC}$ motor with a reducer, a disc and three round magnets ( $\Phi 8 \mathrm{~mm})$ arranged on the disc surface. The transmission gear ratio of the reducer is $15: 1$. The speed of the disc is adjusted by the PLC and the inverter to drive the AC 3-phase motor. The electric connection between the output relay of Y10Y13 of PLC and the forward, reverse, high-speed, low-speed control pins of the inverter is established respectively as shown in Fig. (2). The magnitude of velocity of high-speed, medium-speed and low-speed could be set up and changed through parameters settings of Pr.4, Pr.5, Pr.6. in the inverter to meet different velocity requirements of the moving magnet on the disc.

\subsection{The Tracking Movement of the Hall Sensor}

The Hall sensor's tracking movement is obtained by two servo feed axes in an planar interpolation method. FX3U PLC controller could output its own high pulse or utilize the positioning module FX2N-20GM to control the two axes AC servo units for the tracking movement. Those two ways are described briefly below.

\subsubsection{The Tracking Movement with PLC high Speed Pulse Output}

The output relay of FX3U-64MT PLC is transistor type, which has the ability of generating high-speed pulse output from $\mathrm{Y} 0$ to $\mathrm{Y} 2$, and whose maximum frequency is up to 100 $\mathrm{kHz}$ in biaxial mode. A planar motion can be realized by interpolation mode. Electrical schematic is shown in Fig. (3). The output relay $\mathrm{Y} 0$ and $\mathrm{Y} 4$ are used to control the speed and direction for $\mathrm{X}$-axis $\mathrm{AC}$ servo unit, and the $\mathrm{Y} 1$ and $\mathrm{Y} 5$ for $\mathrm{Y}$-axis AC servo unit, respectively.

\subsubsection{The Tracking Movement with FX2N-20GM Posi- tioning Module}

FX2N-20GM is the two'-axis positioning module for Mitsubishi FX Series PLC, and generates output of two groups of high speed pulse into the two $\mathrm{AC}$ servo units 


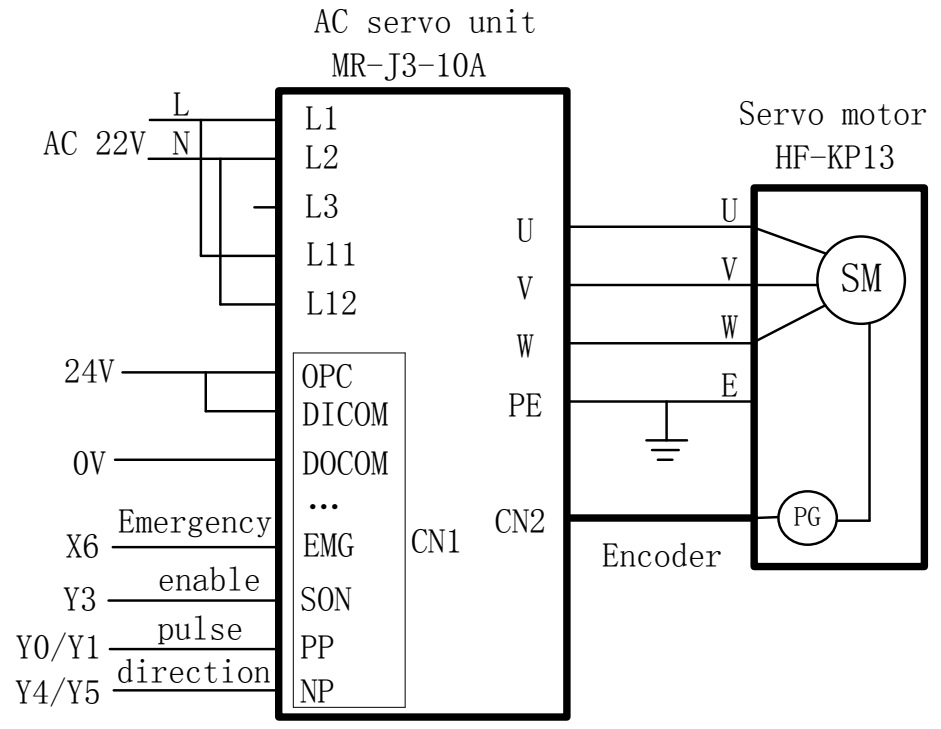

Fig. (3). Electrical connection between the PLC, AC servo unit and servo motor.

to achieve planar movement by interpolation with different speeds. There are four connectors on the panel of FX2N20GM. CON1 is an input or output relay interface, CON2 is a bi-axially external control input interface, while CON3 and CON4 are $\mathrm{X}$ and $\mathrm{Y}$-axis connectors of servo actuating control module units respectively. The CON3 and CON4 are used for the tracking movement of the Hall sensor.

A special visual positioning controller software (VPS) for FX2N-20GM positioning module is utilized to program and achieve a plane line or arc interpolation motion trajectory. Variables parameters during the interpolated motion can be set in the touch screen and the PLC uses the 'TO' instruction to transfer those parameters into the buffer file memory (BFM) in the positioning module FX2N-20GM, and similarly uses the 'FROM' instruction to obtain the operation status information of FX2N-20GM .

\subsection{Synchronous Tracking Control Method}

Synchronous tracking of the moving object involves two processes including, finding the moving target and tracking it. In this tracking system, the moving magnet is placed at various radius on the surface of round disc and the moving trajectories of each magnet form different circles of different radius. The tracking Hall sensor goes to some point on the circle and waits for the moving magnet passing by. When the moving magnet passes through the Hall sensor just below, the Hall sensor indicator light turns on, which shows that the magnet track is found. When the magnet's velocity is low, the servo system can be started directly, which lets the Hall sensor track the moving magnet immediately. If the magnet's velocity is high, the Hall sensor waits for some time. Before the second arrival of the magnet, the tracking Hall sensor starts earlier and gradually accelerates. When the magnet arrives at some point, the Hall sensor is accelerated to approach the speed of the magnet.
The real-time speed adjustments of the Hall sensor are required in order to correct the offset position between the Hall sensor and the circular magnet to maintain a small range of variation during synchronously tracking of the moving magnet. Therefore it is very important to detect real-time magnitude and orientation of the deviation for the tracking system. There may exist many methods to detect the position bias. Linear Hall sensors could be used for this purpose . A single linear Hall sensor could only measure the deviation magnitude but not the orientation of the position offset. Thus at least two linear Hall sensors are required. A Hall switch sensor quantitatively measures neither the offset size nor the orientation of the position offset. However, the utility of multiple switch-type Hall sensors may achieve the detection of the magnitude and the direction of the position offset. Linear Hall sensors cost much higher than switch-type Hall sensors and also have to be equipped with A / D module. therefore, it significantly increases the system cost. A new control method based on two Hall switch-type sensors was presented. These two Hall switch-type sensors were miniaturized and packaged in a $\Phi 8 \mathrm{~mm}$ cylindrical body through custom processing. Thus their appearances seem same as the Hall switch sensor with a diameter $8 \mathrm{~mm}$.

The principle of detecting the position offset between the Hall sensor and the magnet Based on four Hall switch sensors is shown in Fig. (4). In Fig. (4a) the Arabic number indicates the position number of two Hall switch sensors. The relationship between the two Hall sensors and the coordinate system was explicit after these Hall switch sensors were fixed. According to the results of two Hall switch sensors sensing the magnet, the orientation and amplitude of the position offset could be determined qualitatively. In Fig. (4b) the No.1 Hall sensor could not detect the magnet. This indicates that the center of the Hall sensor has been moved over to the $\mathrm{Y}$-axis positive side. Thus it needs to increase the movement speed along the Y-axis negative side until the Hall sensor becomes capable to sense the magnet at the same time so far. The speed value set should be adjusted 


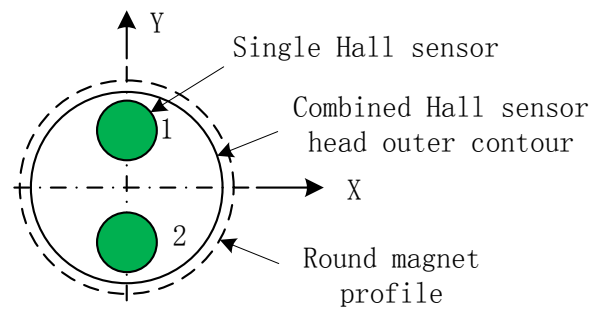

(a) layout of two Hall switch sensors

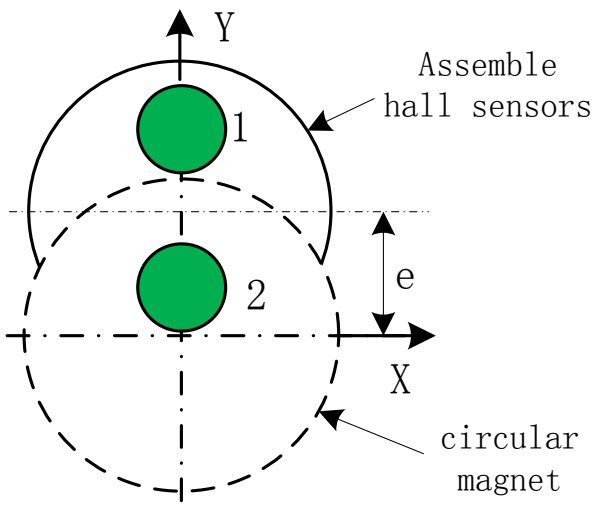

(b) position offset along $\mathrm{Y}$-axis direction

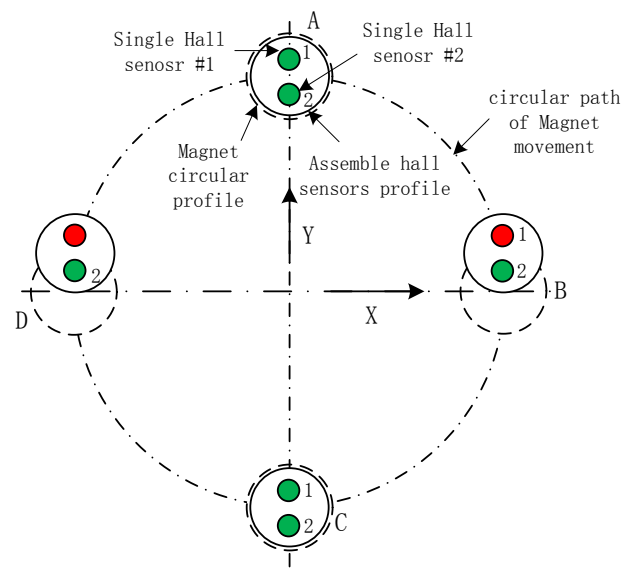

(c) schematic diagram of Hall sensor tracking magnet

Fig. (4). Position offset detection principle with four Hall switch sensors.

according the speed of the moving magnet and actual experimental situations. In Fig. (4c) the schematic diagram of Hall sensor tracking magnet is presented. In the circular path of the magnet in the tracking process, tracking speed of assemble hall sensors could be adjusted only at position B and D. For example, at position $\mathrm{B}$, the assemble hall sensors lagged behind the magnet, therefore, it was required to increase the speed. Similarly at position B, the assembled hall sensors moved the magnet forward, therefore, it decreased the speed.

The offset distance control algorithm based on two Hall switch sensors is shown in Fig. (5). $\mathrm{N}$ in the flow condition judgment block means that the Hall sensor cannot sense the magnet, while $\mathrm{Y}$ can do. For example, $1 \mathrm{~N}$ represents the No. 1 Hall sensor that failed to detect the magnet. The others are similar as $1 \mathrm{~N}$.

\subsection{Control Flow Chart of Tracking System}

PLC received user's setting parameters from the touch screen, such as selection of the magnet tracked, the magnet's rotational speed and direction, the Hall sensor tracking speed's incremental value and so on. Both automatic and 


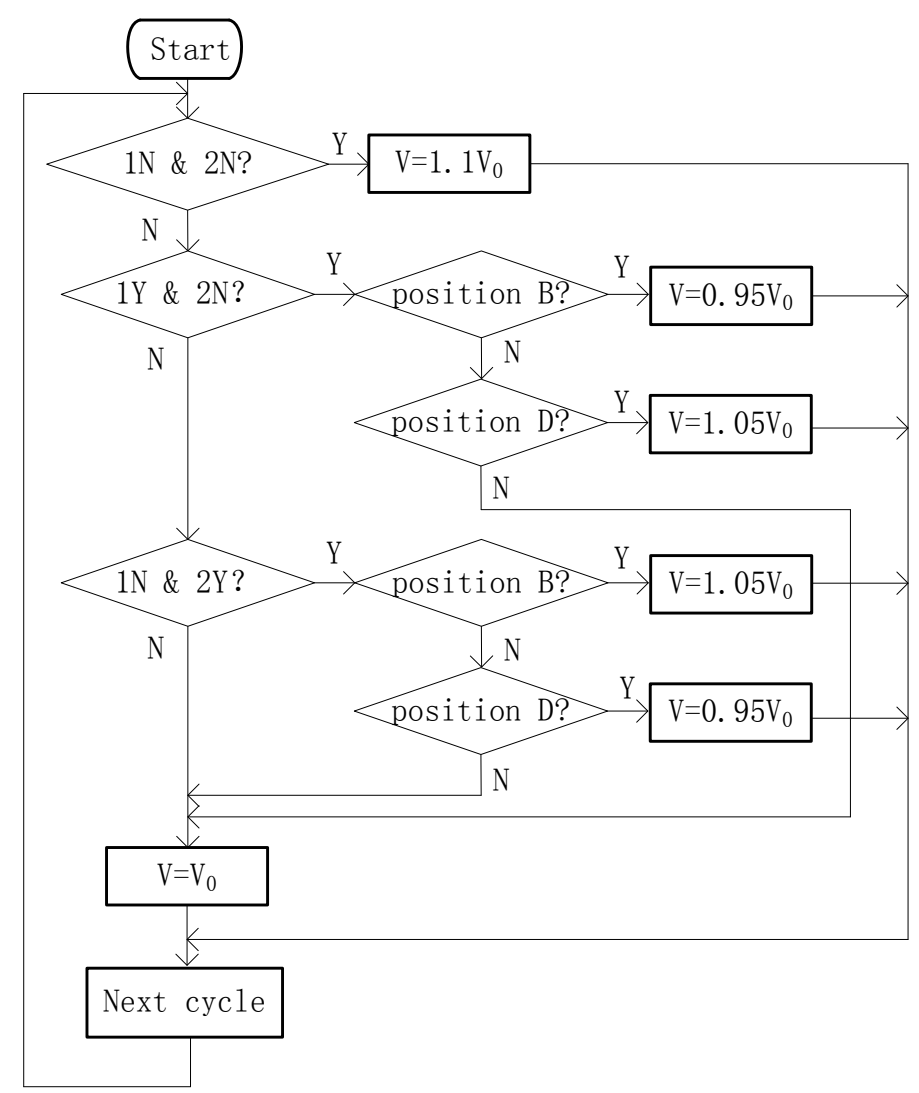

Fig. (5). Tracking control algorithm flow chart.

manual operating modes could be used in PLC controller. PLC control flow chart of program is shown in Fig. (6). The tracking control algorithm illustrated in Fig. 6 is described comprehensively in Fig. (5).

The tracking system of the hall sensor to track the round magnet was established and some tests were conducted. The experimental results showed that when the three-phase AC motor speed is not higher than $1500 \mathrm{rpm}$, the rotation speed of the magnet is not more than $100 \mathrm{rpm}$, and the radius of the tracking magnet is no more than $70 \mathrm{~mm}$. The continuous synchronous tracking could be achieved without separating the Hall sensor and the round magnet. Limited by the tracking system's stiffness, inertia, PLC scan cycle, and highspeed pulse frequency, it is difficult to achieve continuous synchronous tracking at a speed higher than $100 \mathrm{rpm}$.

\subsection{Experimental Process of Synchronous Tracking Sys- tem}

The process of the experiment of synchronous tracking system is mainly consists of four steps. In step 1 initial conditions of the system were set up. These initial conditions mainly include magnet position, magnet speed (ie the frequency of the converter), the numbers of magnets tracked, system's electrical zero position, and manual and automatic mode selection. In step 2, the disc with magnets was made to rotate for creating active movement. Three magnets having the same size with the number $\mathrm{A} \mathrm{B}$ and $\mathrm{C}$ were placed at three positions with different radii of the disc. The synchronous tracking ability of the system could be verified with different radius and speed. In step 3, the biaxial interpolation system was started, and the Hall sensors were actuated to search the magnet and track it synchronously. In step 4, the results of the hall sensor tracking the magnet were detected. Hall sensors detected the magnet and followed it. Since that time, the sensors continuously maintained the induction state with the magnet, which suggested that those two objects achieved synchronous tracking. Otherwise, those two objects would fail to be tracked synchronously.

\section{CONCLUSION}

A synchronous tracking control method which involves using two Hall switch type sensors for detecting the position offset was presented in this paper. This method was proved to be effective by the experiment in which the combined Hall sensor moving based on interpolation principle tried to track the active moving magnet on the disc through PLC. The experimental results show that the Hall sensor could follow the magnets, whose radius is no more than $70 \mathrm{~mm}$, without departing from each other when the rotating speed of the magnets is not more than $100 \mathrm{rpm}$. Though the tracking control speed of this synchronization control method was not very high, obviously for the advantages of simplicity, lowcost and versatility, this method could be provided as technical reference for positional tracking control in some industry application which requires moderate precision and does not require very high speed. 


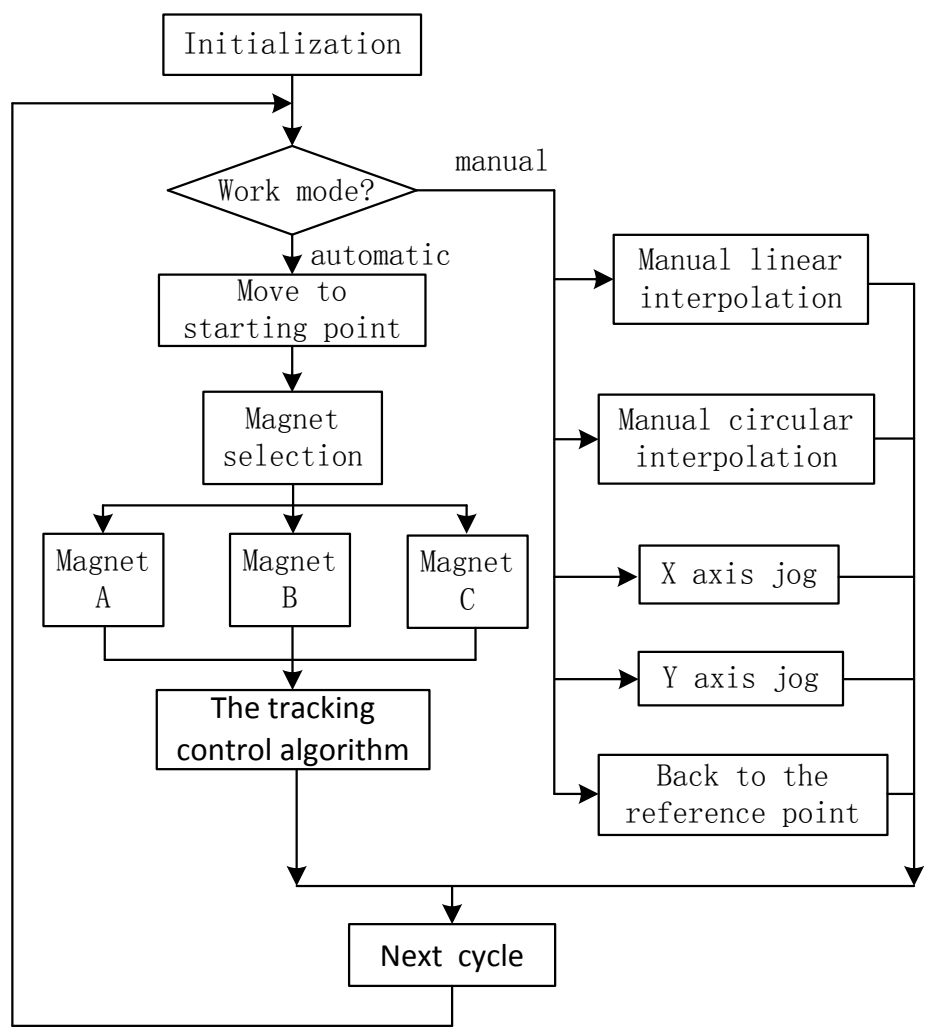

Fig. (6). PLC program flow chart.

\section{CONFLICT OF INTEREST}

The research work was supported by Jiangsu Provincial Department of Education and Jiangsu University of Technology. This article content has no conflict of interest.

\section{ACKNOWLEDGEMENTS}

The work described in this paper was supported by a Postdoctoral Foundation of Jiangsu Province (Grant Nos. 1302023C) and Electrical control and PLC curriculum reform project of Jiangsu University of Technology (Grant Nos. KC13007).

\section{REFERENCES}

[1] R. Li, X. Du, and P. Li, "Design of low-cost acquisition system for multi-channel analog signal for PLC and its application in temperature control system", In: Proceedings of the World Congress on Intelligent Control and Automation (WCICA), pp. 93-98, 2012.

[2] Y. Zhang, P.Q. Yang, F.W. Yuan, and Y.R. Hu, "Design of the advanced automatic door controller based on PLC", Advanced Materials Research, vol. 591-593, pp. 1338-1341, 2012.

[3] J. Hu, Y. Sun, and G. Zhang, "Research on PLC controlling system of servo mechanical presses", In: Proceedings-2013 $5^{\text {th }}$ Conference on Measuring Technology and Mechatronics Automation, ICMTMA, vol. 2013, pp. 919-922, 2013.

[4] X. Wang, "The application of PLC in the Stepper motor closedloop control system design", Advanced Materials Research, vol. 605-607, pp. 1537-1540, 2013

[5] G. Cheng, Z. Liu, and L. Dang, "The roller disassembly and assembly machine controlled by PLC," Applied Mechanics and Materials, vol. 201-202, pp. 99-102, 2012.

[6] Z. Cui, Y. Lv, and X. Zhang, "The design of constant pressure water supply system based on configuration monitoring and PLC control", In: Proceedings of the 2011 International Conference on Electrical, Information Engineering and Mechatronics, vol. 138, pp. 945-952, 2012.

[7] Yousif I., Al Mashhadany. "Design and Implement of a Programmable Logic Controller (PLC) for Classical Control Laboratory". Intelligent Control and Automation, vol. 3, no. 1, pp. 44-49, 2012.

[8] M.H. Rahman, C. Ochoa-Luna, J. Rahman, M. Saad, and P Archambault "Force-position control of a robotic exoskeleton to provide upper extremity movement assistance", International Journal of Modelling, Identification and Control, vol. 21, no. 4, pp. 390-400, 2014

[9] Y. Zhang, T. Yang, C. Li, S. Liu, C. Du, M. Li, and H. Sun, "Fuzzy-PID control for the position loop of aerial inertially stabilized platform", Aerospace Science and Technology, vol. 36, no. 7, pp. 21-26, 2014.

(C) Shangrong et al.; Licensee Bentham Open.

This is an open access article licensed under the terms of the Creative Commons Attribution Non-Commercial License (http://creativecommons.org/licenses/by-nc/4.0/) which permits unrestricted, non-commercial use, distribution and reproduction in any medium, provided the work is properly cited. 